\title{
Finite-Amplitude Standing Acoustic Waves in a Cubically Nonlinear Medium
}

\author{
O. V. Rudenko ${ }^{a}$, C. M. Hedberg ${ }^{b}$, and B. O. Enflo ${ }^{c}$ \\ ${ }^{a}$ Faculty of Physics, Moscow State University, Vorob'evy gory, Moscow, 119992 Russia \\ e-mail: rudenko@acs366.msu.ru \\ ${ }^{b}$ School of Engineering, Blekinge Institute of Technology, S-37179 Karlskrona, Sweden \\ e-mail: claes.hedberg@bth.se \\ ${ }^{c}$ Department of Mechanics, Royal Institute of Technology, S-10044 Stockholm, Sweden \\ e-mail:benflo@mech.kth.se \\ Received February 26, 2007
}

\begin{abstract}
The acoustic field in a resonator filled with a cubically nonlinear medium is investigated. The field is represented as a linear superposition of two strongly distorted counterpropagating waves. Unlike the case of a quadratically nonlinear medium, the counterpropagating waves in a cubically nonlinear medium are coupled through their mean (over a period) intensities. Free and forced standing waves are considered. Profiles of discontinuous oscillations containing compression and expansion shock fronts are constructed. Resonance curves, which represent the dependences of the mean field intensity on the difference between the boundary oscillation frequency and the frequency of one of the resonator modes, are calculated. The structure of the profiles of strongly distorted "forced" waves is analyzed. It is shown that discontinuities are formed only when the difference between the mean intensity and the detuning takes certain negative values. The discontinuities correspond to the jumps between different solutions to a nonlinear integro-differential equation, which, in the case of small dissipation, degenerates into a third-degree algebraic equation with an undetermined coefficient. The dependence of the intensity of discontinuous standing waves on the frequency of oscillations of the resonator boundary is determined. A nonlinear saturation is revealed: at a very large amplitude of the resonator wall oscillations, the field intensity in the resonator ceases depending on the amplitude and cannot exceed a certain limiting value, which is determined by the nonlinear attenuation at the shock fronts. This intensity maximum is reached when the frequency smoothly increases above the linear resonance. A hysteresis arises, and a bistability takes place, as in the case of a concentrated system at a nonlinear resonance.
\end{abstract}

PACS numbers: 43.20.Ks, 43.25.-x, 43.25.Gf

DOI: $10.1134 / \mathrm{S} 1063771007040057$

\section{INTRODUCTION}

Intense acoustic waves in resonators filled with weakly nonlinear dissipative media have been studied in detail only for quadratically nonlinear media. Such a nonlinearity is known to be dominant for acoustic waves in fluids and for longitudinal waves in solids. Reviews of the results obtained for quadratically nonlinear resonators can be found in [1-3].

As for cubically nonlinear systems, they were rather poorly investigated. The study of these systems is of interest for two reasons.

First, this is a new physical object, whose evolution radically differs from that of a quadratically nonlinear wave. This new type of evolution was studied in terms of the general theory of nonlinear waves in nondispersive media by Lee-Bapty and Crighton for plane waves [4] and by Rudenko and Sapozhnikov [5] for non-onedimensional wave beams. A modified Burgers equation for the cubically nonlinear case was derived by Zabolotskaya [6] as a model equation for a specific physical situation. This derivation shows that transverse elastic waves in a homogeneous solid obey a cubically nonlinear wave equation, while the quadratic nonlinearity proves to be vanishingly small. The relation between the elastic constants and the cubic nonlinearity coefficient for transverse waves was corrected by us in [7].

Second, cubically nonlinear media attract considerable interest in connection with new applied problems. One group of such problems is concerned with the excitation of intense shear waves for medical purposes [5, $8,9]$, and the other, with various geophysical applications [10]. Experimental data on cubically nonlinear properties of such media as rubber or phantom biological tissue were reported in [11].

\section{METHOD FOR SIMPLIFYING THE PROBLEM}

The success in studying standing waves in quadratically nonlinear resonators was achieved by using the approximation based on considering two nonlinear counterpropagating waves. A detailed explanation and the physical foundation of this approach can be found in $[2,12]$. 
A similar approach can be used for standing waves in a cubically nonlinear resonator. However, in this case, the model of completely independent counterpropagating waves fails.

To show how the model can be modified and how the approach used in [2] can be applied in our case, we consider a wave equation describing transverse waves in a homogeneous solid [13-15]:

$$
\frac{\partial^{2} u}{\partial x^{2}}-\frac{1}{c^{2}} \frac{\partial^{2} u}{\partial t^{2}}=-\frac{2 \varepsilon}{3 c^{4}} \frac{\partial^{2} u^{3}}{\partial t^{2}} .
$$

Here, $u$ is the particle velocity, $\varepsilon$ is the acoustic nonlinearity parameter, and $c$ is the velocity of wave propagation. Equation (1) is the simplest second-order differential equation containing a cubic nonlinearity. Applying the slowly varying profile approach [13], from Eq. (1), we derive a simplified first-order evolution equation

$$
\frac{\partial u}{\partial x}-\frac{\varepsilon}{c^{3}} u^{2} \frac{\partial u}{\partial \tau}=0
$$

for simple (Riemann) waves traveling in a cubically nonlinear medium. Here, $\tau=t-x / c$ and $x$ is the "slow" coordinate [13].

The properties of cubic Riemann waves were studied in [4]. It was shown that a progressive nonlinear distortion of an initially harmonic wave leads to the formation of an asymmetric temporal profile. A further propagation leads to the formation of profile discontinuities. Unlike a quadratically nonlinear wave, which only contains pressure shocks, each of the periods of the cubic wave contains both a compression shock and an expansion shock. A cubic nonlinearity affects the propagation velocity; for acoustic beams, such a velocity variation in the transverse cross section may give rise to the selffocusing or defocusing of beams $[5,14,15]$.

We seek the solution to Eq. (1) in the form

$$
\begin{gathered}
u=u_{+}\left(x_{1}=\mu x, \tau_{+}=t-\frac{x}{c}\right) \\
+u_{-}\left(x_{1}=\mu x, \tau_{-}=t+\frac{x}{c}\right) .
\end{gathered}
$$

Here, $\mu \ll 1$ is the small parameter of the problem; for a cubically nonlinear wave, it is on the order of the square of the acoustic Mach number $\left(\sim \varepsilon u_{\max }^{2} / c^{2}\right)$. Substituting Eq. (3) into Eq. (1) and ignoring the terms on the orders of $\mu^{2}$ and $\mu^{3}$, we obtain

$$
\begin{gathered}
\left(-\frac{2 \varepsilon}{3 c^{4}}\right)^{-1}\left(-\frac{2}{c} \frac{\partial^{2} u_{+}}{\partial x \partial \tau_{+}}+\frac{2}{c} \frac{\partial^{2} u_{-}}{\partial x \partial \tau_{-}}\right)=\frac{\partial^{2} u_{+}^{3}}{\partial \tau_{+}^{2}}+3 \frac{\partial^{2} u_{+}^{2}}{\partial \tau_{+}^{2}} u_{-} \\
+3 u_{+}^{2} \frac{\partial^{2} u_{-}}{\partial \tau_{-}^{2}}+6 \frac{\partial u_{+}^{2}}{\partial \tau_{+}} \frac{\partial u_{-}}{\partial \tau_{-}}+3 \frac{\partial^{2} u_{+}}{\partial \tau_{+}^{2}} u_{-}^{2} \\
+3 u_{+} \frac{\partial^{2} u_{-}^{2}}{\partial \tau_{-}^{2}}+6 \frac{\partial u_{+}}{\partial \tau_{+}} \frac{\partial u_{-}^{2}}{\partial \tau_{-}}+\frac{\partial^{2} u_{-}^{3}}{\partial \tau_{-}^{2}} .
\end{gathered}
$$

Let $u_{+}$and $u_{-}$be rapidly oscillating functions of variables $\tau_{+}$and $\tau_{-}$, respectively. Let the period-average values of these functions be equal to zero: $\left\langle u_{+}\right\rangle_{\tau_{+}}=$ $\left\langle u_{-}\right\rangle_{\tau_{-}}=0$. By sequentially averaging the above expression over the variables $\tau_{-}$and $\tau_{+}$, we arrive at the system of equations

$$
\begin{aligned}
& \frac{\partial u_{+}}{\partial x}-\frac{\varepsilon}{c^{3}}\left(\left\langle u_{-}^{2}\right\rangle+u_{+}^{2}\right) \frac{\partial u_{+}}{\partial \tau_{+}}=0, \\
& \frac{\partial u_{-}}{\partial x}+\frac{\varepsilon}{c^{3}}\left(\left\langle u_{+}^{2}\right\rangle+u_{-}^{2}\right) \frac{\partial u_{-}}{\partial \tau_{-}}=0 .
\end{aligned}
$$

These equations, unlike the corresponding equations for a quadratically nonlinear medium, are not independent. They are coupled through the averaged squares of the variables $u_{+}$and $u_{-}$, i.e., through the mean intensities $I_{+}=\left\langle u_{+}^{2}\right\rangle \neq 0$ and $I_{-}=\left\langle u_{-}^{2}\right\rangle \neq 0$. For standing waves, we have $I_{+}=I_{-}=I$.

Using the method described in $[2,12]$, one can easily verify that all of the omitted terms are nonresonant and cannot noticeably affect the energy transfer between the harmonics of the two counterpropagating waves.

The nonlinear field in the resonator can be represented as a sum of two exact solutions to Eqs. (4) and (5), as in the case of the corresponding representation for the problem with quadratic nonlinearity. We write these two solutions in the following convenient form:

$$
\begin{aligned}
& u_{+}=F_{+}\left[\omega t-k(x-L)+\frac{\varepsilon}{c^{2}} k(x-L)\left(I+u_{+}^{2}\right)\right], \\
& u_{-}=F_{-}\left[\omega t+k(x-L)-\frac{\varepsilon}{c^{2}} k(x-L)\left(I+u_{-}^{2}\right)\right] .
\end{aligned}
$$

Here, $k=\omega / c$ is the wave number and $x=L$ is the coordinate of the right-hand boundary of the nonlinear medium occupying the region $0<x<L$. The functions $F_{+}$and $F_{-}$ are determined from the boundary conditions.

In a cubically nonlinear medium, the field excitation by a harmonic source of frequency $\omega$ is known to lead to the excitation of only odd harmonics with frequencies $(2 n+1) \omega$. If $\omega$ is close to the lowest mode frequency $\omega_{1}$, a pure standing wave can be formed only when the higher harmonic frequencies are close to the frequencies of the respective higher modes. This regime is of most interest, because it allows the accumulation of a considerable amount of energy in the resonator even with a weak external source. The necessary spectrum

$\omega_{2 n+1}=(2 n+1) \omega_{1}, \quad \omega_{1}=\frac{\pi c}{2 L}, \quad n=0,1,2 \ldots(7)$

is characteristic of a resonator whose one wall (e.g., $x=0$ ) is rigid with $u(x=0, t)=0$, while the other wall corresponds to the maximum particle velocity, i.e., 


$$
\left.\frac{\partial u}{\partial x}\right|_{x=L}=\left(\frac{\partial u_{+}}{\partial x}+\frac{\partial u_{-}}{\partial x}\right)_{x=L}=0 .
$$

From boundary condition (8), we obtain that the arbitrary functions $F_{+}$and $F_{-}$involved in solution (6) should be identical: $F_{+}=F_{-}=F$. Then, the field in the resonator takes the form

$$
\begin{gathered}
u=F\left[\omega t-\frac{\omega}{c}(x-L)+\frac{\varepsilon \omega}{c^{3}}\left(I+F^{2}\right)(x-L)\right] \\
+F\left[\omega t+\frac{\omega}{c}(x-L)-\frac{\varepsilon \omega}{c^{3}}\left(I+F^{2}\right)(x-L)\right] .
\end{gathered}
$$

Let the boundary $x=0$ oscillate according to the harmonic law

$$
u=(x=0, t)=A \sin (\omega t) .
$$

Using Eq. (10), we reduce Eq. (9) to the nonlinear functional equation

$$
\begin{gathered}
F\left[\omega t+k L-\frac{\varepsilon}{c^{2}} k L\left(I+F^{2}\right)\right] \\
+F\left[\omega t-k L+\frac{\varepsilon}{c^{2}} k L\left(I+F^{2}\right)\right]=A \sin (\omega t) .
\end{gathered}
$$

Linearizing Eq. (11) by formally setting $\varepsilon=0$, we obtain its general solution

$$
\begin{gathered}
F=\frac{A \sin (\omega t)}{2 \cos (k L)} \\
+\sum_{n=0}^{\infty}\left[A_{2 n+1} \cos (2 n+1) \omega_{1} t+B_{2 n+1} \sin (2 n+1) \omega_{1} t\right] .
\end{gathered}
$$

Exactly at resonance, for example, at $\omega=\omega_{1}$, solution (12) describes oscillations linearly increasing with time without bound:

$$
F=-\frac{A}{\pi}\left(\omega_{1} t\right) \cos \left(\omega_{1} t\right)
$$

This growth (as it is known) is limited by absorption, nonlinearity, or frequency detuning from the exact resonance:

$$
k L=\frac{\pi}{2}+\Delta, \quad \Delta=\left(\omega-\omega_{1}\right) \frac{L}{c}=\frac{\pi}{2} \frac{\left(\omega-\omega_{1}\right)}{\omega_{1}} \ll 1 .
$$

Here and below, the dimensionless detuning $\Delta$ is assumed to be small. If $\Delta \neq 0$ is taken into account, the oscillations prove to be modulated and described by a function limited in time:

$$
F=-A \sin ^{-1}\left(\frac{\pi}{2} \frac{\omega-\omega_{1}}{\omega_{1}}\right) \sin \left(\frac{\omega-\omega_{1}}{2} t\right) \cos \left(\frac{\omega+\omega_{1}}{2} t\right) .
$$

Using the method described in [2], we reduce functional equation (8) to the differential equation

$$
\frac{\partial U}{\partial T}+\left(\Delta-\frac{\pi \varepsilon}{2} J-\frac{\pi \varepsilon}{2} U^{2}\right) \frac{\partial U}{\partial \xi}-D \frac{\partial^{2} U}{\partial \xi^{2}}=-\frac{M}{2} \cos \xi
$$

Here, for simplicity and for better comparison of the results, we used the same dimensionless notation as in [2]:

$$
\begin{gathered}
U=\frac{F}{c}, \quad M=\frac{A}{c}, \quad J=\frac{I}{c^{2}}, \\
\xi=\omega t+\frac{\pi}{2}, \quad T=\frac{\omega t}{\pi}, \quad D=\frac{b \omega^{2}}{2 c^{3} \rho} L .
\end{gathered}
$$

The dissipative term with the second derivative was introduced into Eq. (16) according to the procedure described in [2]. The parameter $D$ is also a small quantity; it is proportional to the effective viscosity $b$ and equal to the product of the wave absorption coefficient by the resonator length $L$. In Eq. (16), we used two time variables: the "fast" time $\xi$ and the "slow" time $T$. The "slowness" of $T$ is determined by the smallness of the three coefficients involved in Eq. (16), namely, $\Delta, D$, and $M \sim U$ (see also [2]).

\section{FREE STANDING WAVES}

It is expedient to begin the analysis of standing waves by considering the simplest case of free oscillations. Let the left-hand boundary of the resonator be immobile, so that the particle velocity at this boundary is equal to zero: $u(x, t)=0$ at $x=0$. At the right-hand boundary $x=L$, the derivative of the particle velocity is zero: $\partial u / \partial x=0$. At the initial instant $t=0$, large-amplitude oscillations arise between the stationary walls. The subsequent evolution of the acoustic field occurs without any additional influx of energy from the outside. The problem is solved using the approach described in Section 2. Instead of inhomogeneous equation (16) for the auxiliary function $U$, we will solve a homogeneous $(M=0)$ equation. The results of analyzing the homogeneous equation corresponding to Eq. (16) are known; they were described in detail in $[4,5]$ for propagating perturbations. However, as it was demonstrated above, it is possible to construct a standing wave as a sum of two counterpropagating waves. This procedure is illustrated in Fig. 1.

One can see that each of the two initially harmonic waves acquires a sawtooth shape within a sufficiently long time. Unlike the case of a quadratically nonlinear medium, every "tooth" (half-period) has a trapezoidal shape rather than a triangular one. The peak of the positive particle velocity at the compression shock front is twice as large as the magnitude of the negative velocity at this shock front. On the other hand, the magnitude of the negative velocity at the expansion shock front is twice as large as the positive velocity value at the same shock front.

Using the representation in the form of a linear superposition of two waves strongly distorted by the 


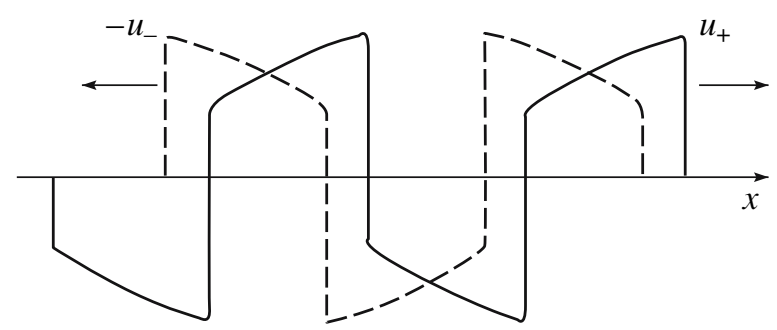

Fig. 1. Shapes of the wave profiles of counterpropagating waves in a cubically nonlinear medium.

cubic nonlinearity, we construct the standing wave profiles according to the formula following from Eq. (9):

$$
\frac{u}{c}=U[\omega t-k(x-L)]+U[\omega t+k(x-L)] .
$$

Here, $U$ describes the distorted profile of the wave traveling in the positive or negative direction of the $x$ axis. The arguments of both functions $U$ in Eq. (18) contain no nonlinear terms, because the profiles are constructed within a single period of oscillations. Here, it should be noted that nonlinear effects can accumulate and considerably distort the profile on the interval extending over many periods; their number is proportional to $\left(\varepsilon M^{2}\right)^{-1} \gg 1$ (see, e.g., [12]).

The temporal profiles measured in different cross sections of the resonator $(L / 4, L / 2$, and $L)$ are shown in Fig. 2. One can see how two narrow velocity "pulses" (the positive and negative ones) are formed as the cross section approaches the left-hand end of the resonator. Exactly at the wall $x=0$, these peaks vanish and the velocity becomes identically equal to zero.

\section{FORCED STANDING WAVES}

In contrast to the homogeneous equation, the complete version of inhomogeneous equation (16) has not been investigated, and no corresponding results have been obtained for traveling waves to use them as the basis for describing standing waves.

The first attempt to analyze the equation employs the simplest "harmonic balance" method, which is well known from the theory of oscillations. According to this method, a weakly nonlinear solution to Eq. (16) should be sought in the form

$$
U=A(T) \cos \xi+B(T) \sin \xi .
$$

Separating the terms multiplying the functions $\sin \xi$ and $\cos \xi$, we obtain a system of two coupled ordinary differential equations:

$$
\begin{gathered}
\frac{d B}{d T}-\left[\Delta-\frac{3 \pi \varepsilon}{8}\left(A^{2}+B^{2}\right)\right] A+D B=0, \\
\frac{d A}{d T}+\left[\Delta-\frac{3 \pi \varepsilon}{8}\left(A^{2}+B^{2}\right)\right] B+D A=-\frac{M}{2} .
\end{gathered}
$$

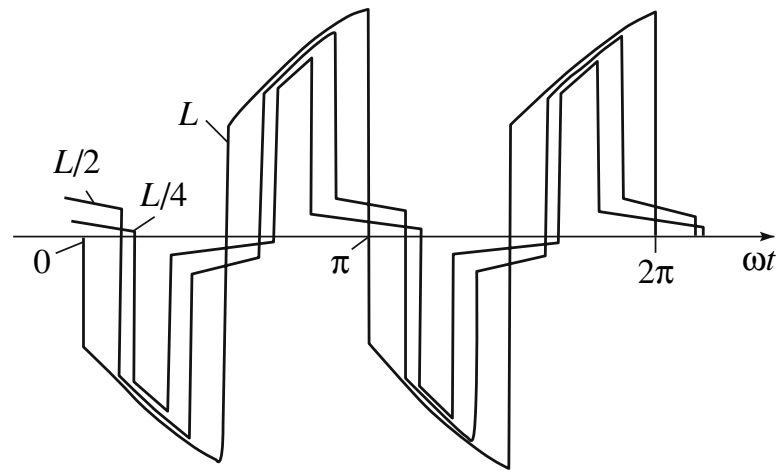

Fig. 2. Shape of a standing wave in time: measurements in different cross sections of the resonator at distances of $L / 4$, $L / 2$, and $L$ from the left-hand end of the resonator.

This system can easily be analyzed by numerical methods. However, it has an analytical solution that corresponds to steady-state oscillations. This solution is obtained for $T \longrightarrow \infty$, when the time derivatives in Eq. (20) become equal to zero: $d A / d T=d B / d T=0$. The steady-state solution to Eqs. (20) is as follows:

$$
\left[\Delta-\frac{3 \pi \varepsilon}{8}\left(A^{2}+B^{2}\right)\right]^{2}+D^{2}=\frac{M^{2}}{4}\left(A^{2}+B^{2}\right)^{-1} .
$$

This solution can be represented in the simplest form as

$$
\delta_{1}=y \pm \sqrt{y^{-1}-d^{2}}
$$

where

$$
\begin{gathered}
\delta_{1}=\frac{\Delta}{C}, \quad d=\frac{D}{C}, \quad y=\frac{3}{4} \pi \varepsilon \frac{J}{C}, \\
C=\left(\frac{3 \pi \varepsilon M^{2}}{32}\right)^{1 / 3}, \quad J=\frac{1}{2}\left(A^{2}+B^{2}\right) .
\end{gathered}
$$

The resonance curves for the mean intensity $y\left(\delta_{1}\right)$ are shown in Fig. 3 for different values of the dissipation parameter: $d^{2}=2,1.25,0.75,0.5$, and 0.4 . As the absorption decreases, the shape of the curve representing the frequency response is distorted. In the case of a weak absorption $d<\sqrt{3} / 2$, this curve describes an ambiguous function in a certain region of positive values of detuning $\delta_{1}$.

\section{FORCED WAVES WITH DISCONTINUITIES}

Solution (21) was obtained using the harmonic balance method (19). Its accuracy is low for strongly distorted waves, especially in the most interesting case of a wave profile with shock fronts. Therefore, we consider the forced waves described by Eq. (16) by using another approach. The steady-state regime achieved in 


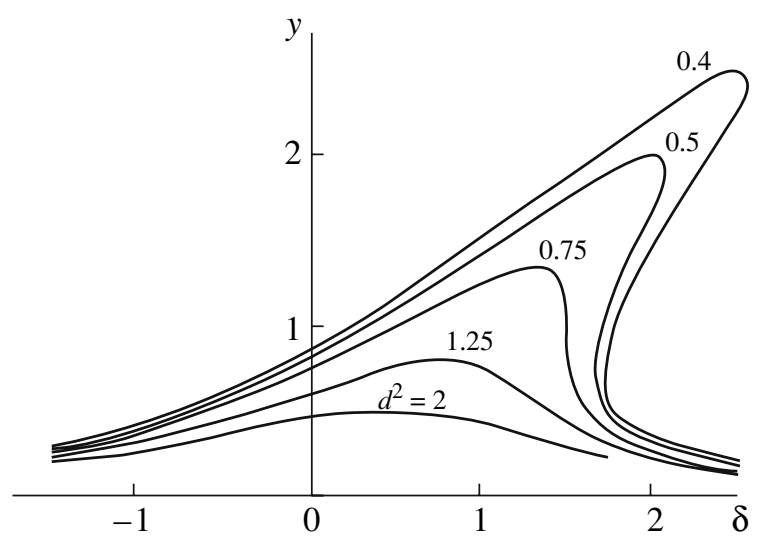

Fig. 3. Resonance curves for the mean intensity of oscillations in a cubically nonlinear resonator for different values of the dissipation parameter $d$.

the limiting case $T \longrightarrow \infty$ is described by the ordinary differential equation following from Eq. (16):

$$
\left(\Delta-\frac{\pi \varepsilon}{2} J-\frac{\pi \varepsilon}{2} U^{2}\right) \frac{d U}{d \xi}-D \frac{d^{2} U}{d \xi^{2}}=-\frac{M}{2} \cos \xi .
$$

The integration of Eq. (24) with allowance for the condition $\langle U\rangle=0$ yields the first-order equation

$$
D \frac{d U}{d \xi}+\frac{\pi \varepsilon}{6} U^{3}+\left(\frac{\pi \varepsilon}{2} J-\Delta\right) U=\frac{M}{2} \sin \xi .
$$

To simplify further calculations, we introduce the notations

$$
\begin{gathered}
V=U\left(3 \frac{M}{\pi \varepsilon}\right)^{-1 / 3}, \quad j=J\left(3 \frac{M}{\pi \varepsilon}\right)^{-2 / 3}, \\
\Gamma=D\left(\frac{\pi \varepsilon}{24} M^{2}\right)^{-1 / 3}, \quad \delta=\frac{\Delta}{3}\left(\frac{\pi \varepsilon}{24} M^{2}\right)^{-1 / 3},
\end{gathered}
$$

which slightly differ from notations (23) introduced earlier. Specifically, the mean intensities $j$ and $y$ only differ in a numerical factor of about unity; a similar difference takes place for other pairs: the dimensionless dissipative coefficients $\Gamma$ and $d$ and the normalized detunings $\delta$ and $\delta_{1}$. These differences follow from simplicity considerations. Notations (23) allow us to represent solution (21) to Eqs. (20) in simplest form (22), whereas notations (26) allow us to reduce Eq. (25) to the form

$$
\Gamma \frac{d V}{d \xi}+V^{3}+3(j-\delta) V=\sin \xi .
$$

It is evident that weakly absorbing media are of most interest from the physical point of view, because nonlinear phenomena in such media can be clearly pronounced. To describe the wave profile in an ideal nondissipative medium, we set $\Gamma=0$ in Eq. (27). As is demonstrated below, the approximation $\Gamma \longrightarrow 0$ is valid everywhere except for the small vicinities of the shock

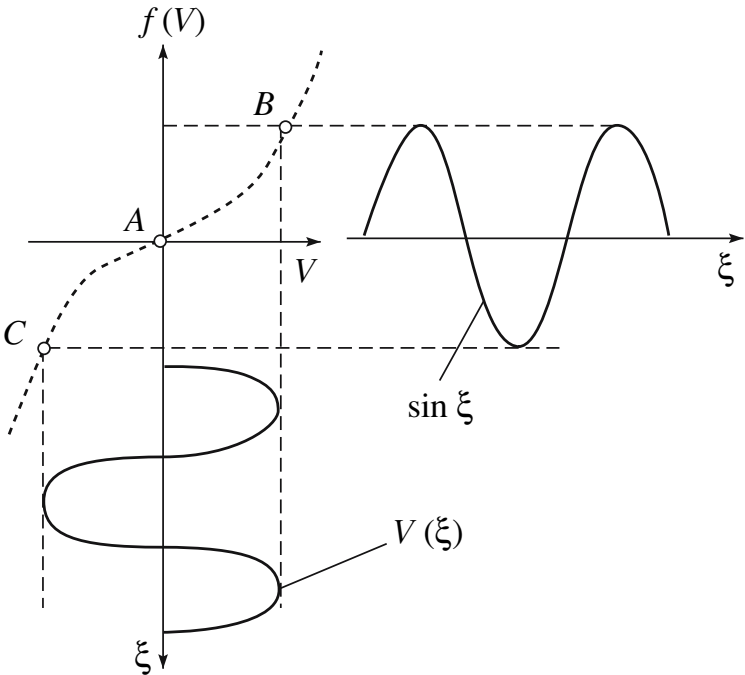

Fig. 4. Graphical analysis of the wave profile. The $f(V)$ curve is shown by the dashed line, along which the vertical projection of the function $\sin \xi$ moves. The motion begins at the instant $\xi=0$ at point $A$; then, oscillations between points $B$ and $C$ take place.

fronts. This approximation corresponds to neglecting the derivative, which results in the transformation of differential equation (27) into an algebraic equation. However, this new equation

$$
f(V)=V^{3}+3(j-\delta) V=\sin \xi
$$

is not a simple cubic equation, because its solution should satisfy additional integral conditions:

$$
\langle V\rangle=\frac{1}{2 \pi} \int_{0}^{2 \pi} V d \xi=0, \quad\left\langle V^{2}\right\rangle=\frac{1}{2 \pi} \int_{0}^{2 \pi} V^{2} d \xi=j .
$$

Hence, the constant $j$ involved in Eq. (28) is not known in advance; it should only be determined after finding the solution to Eq. (28) with an arbitrary value of $j$.

Let us separately analyze different possible situations.

(a) Let the mean intensity exceed the detuning, i.e., $j-\delta \equiv a^{2}>0$.

In this case, Eq. (28) takes the form

$$
f(V)=V^{3}+3 a^{2} V=\sin \xi .
$$

Qualitatively, the behavior of the solution is shown in Fig. 4. The shape of the solution in time, which represents the wave profile, is determined graphically. First, according to the equation $f(V)=\sin \xi$, the imaging point is found; as the time $\xi$ increases, this point moves along the curve $f(V)$ over the cycle $A \longrightarrow B \longrightarrow C \longrightarrow A$. Second, the "horizontal" projection of motion is plotted for this point; the projection gives the wave profile $V(\xi)=f^{-1}[V(\xi)]$, where $f^{-1}$ is the function that is the inverse of the function $f$. 


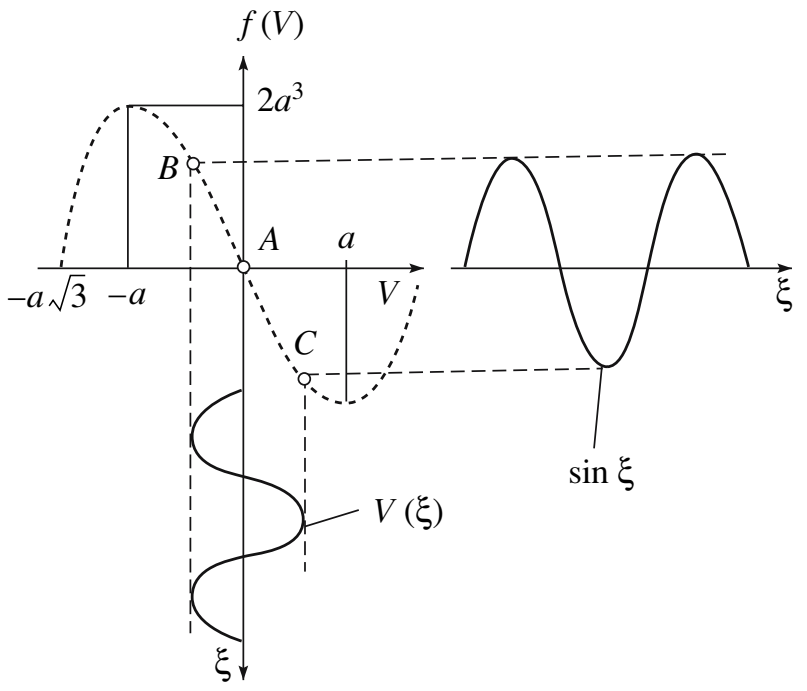

Fig. 5. Graphical analysis of the wave profile (by analogy with Fig. 4) for large positive values of detuning $\delta=j+a^{2}$ in the case of $2 a^{3}>1$.

One can see that, in this case, the profile $V(\xi)$ has no singularities, because the function $f(V)$ is monotonic. The period and polarity of $V(\xi)$ are the same as those of the right-hand side of Eq. (30), which has the form of $\sin \xi$. Evidently, the wave $V(\xi)$ is distorted; its spectrum contains higher harmonics, because the plot of the function $f(V)=V^{3}+3 a^{2} V$ is not a straight line. The difference between $V(\xi)$ and $\sin \xi$, which manifests itself in the nonlinear distortion of the wave, increases with an increase in the oscillation amplitude.

(b) Let the mean intensity be equal to the detuning, i.e., $j-\delta=0$.

For this case, Eq. (28) takes the form

$$
f(V)=V^{3}=\sin \xi .
$$

It has an exact analytical solution:

$$
\begin{gathered}
V=\sin ^{1 / 3}(\xi)=\sum_{n=1}^{\infty} B_{2 n-1} \sin [(2 n-1) \xi], \\
j=\frac{1}{\sqrt{\pi}} \Gamma\left(\frac{5}{6}\right) \approx 0.64 .
\end{gathered}
$$

The Fourier series expansion only contains the odd harmonics with the amplitudes

$$
\begin{gathered}
B_{2 n-1}=\frac{3}{2^{4 / 3}} \frac{\Gamma\left(\frac{7}{3}\right)}{\Gamma\left(\frac{2}{3}+n\right) \Gamma\left(\frac{5}{3}-n\right)}, \\
B_{2 n}=0, \quad n=1,2,3, \ldots
\end{gathered}
$$

The even harmonics are absent in this expansion, because the nonlinearity of the medium is cubic rather than quadratic.

The numerical estimate in Eqs. (32) indicates the upper limit for the case $j-\delta>0$. Evidently, an increase in the detuning within the interval $-\infty<\delta<0.64$ leads to an increase in the mean intensity from zero to 0.64 .

(c) Let the positive detuning exceed the mean intensity, i.e., $j-\delta \equiv-a^{2}<0$, and, in addition, $2 a^{3}>1$.

Then, Eq. (28) takes the form

$$
f(V)=V^{3}-3 a^{2} V=\sin \xi .
$$

The behavior of the solution to Eq. (34) is analyzed in Fig. 5. One can see that the oscillations of the righthand side of Eq. (34) lead to the motion of the imaging point over the cycle $A \longrightarrow B \longrightarrow C \longrightarrow A$ along the curve $f(V)$. As in case (a), the wave profile $V(\xi)$ is distorted by the nonlinearity but contains no discontinuities. The polarity of oscillations of the function $V(\xi)$ is inverse with respect to $\sin \xi$, unlike the profile shown in Fig. 4.

(d) Let the positive detuning exceed the mean intensity, $j-\delta \equiv-a^{2}<0$, but $2 a^{3}<1$.

This case is most difficult to analyze because of the discontinuities arising in different parts of the wave profile.

Let us consider Eq. (34) under the assumption that $0<2 a^{3}<1$. In this case, algebraic equation (34) has one real root for $|\sin \xi|>2 a^{3}$ and three real roots for $|\sin \xi|<$ $2 a^{3}$. Assuming that $2 a^{3}<\sin \xi<1$, we determine the single root $V_{1}$ :

$$
\begin{gathered}
V_{1}=\sqrt[3]{0.5 \sin \xi+0.5 \sqrt{\sin ^{2} \xi-4 a^{6}}} \\
+\sqrt[3]{0.5 \sin \xi-0.5 \sqrt{\sin ^{2} \xi-4 a^{6}}} .
\end{gathered}
$$

For the case $0<\sin \xi<2 a^{3}$, we determine three real roots:

$$
\begin{gathered}
V_{1}=2 a \cos f(\xi), \quad f(\xi) \equiv \frac{1}{3} \arccos \frac{\sin \xi}{2 a^{3}}, \\
V_{2}=-a \cos f(\xi)-\sqrt{3} a \sin f(\xi), \\
V_{3}=-a \cos f(\xi)+\sqrt{3} a \sin f(\xi) .
\end{gathered}
$$

From Eq. (34), it follows that the solution should change its sign when the function $\sin \xi$ becomes negative.

To obtain a qualitative graphical representation of the wave profile, we consider Fig. 6. Let the motion begin at the point $A$ with the value $V=0$ at $\xi=0$. From Eqs. (36)-(38), we obtain that, at $\xi=0$, three roots are present: $V_{1}=a \sqrt{3}, V_{2}=-a \sqrt{3}$, and $V_{3}=0$. It is evident that, for the argument $\xi$ increasing in the region $0 \leq \xi<\xi_{S}$, where $\sin \xi_{S}=2 a^{3}$, we should choose the third root $V=V_{3}$. 
When the argument reaches the value $\xi=\xi_{S}$, the solution varies in a jump-like manner from $V=$ $V_{3}\left(\xi_{S}\right)=-a$ to $V=V_{1}\left(\xi_{S}\right)=2 a$, which corresponds to the jump from position $B$ to position $C$. Within the segment $C D$, only one real solution is present: $V=V_{1}$ given by Eq. (35). It reaches its maximum at point $D$ at $\xi=\pi / 2$ :

$$
\begin{gathered}
V(\pi / 2)=V_{1}(\pi / 2) \\
=\sqrt[3]{0.5+0.5 \sqrt{1-4 a^{6}}}+\sqrt[3]{0.5-0.5 \sqrt{1-4 a^{6}}} .
\end{gathered}
$$

As the variable $\xi$ increases further, it passes through the value $\xi=\pi-\xi_{S}$, and the solution $V_{1}$ changes its analytical representation from Eq. (35) to Eq. (36).

At $\xi=\pi$, the function $\sin \xi$ changes sign and, as follows from Eq. (34), the root $V_{1}$ should change from $a \sqrt{3}$ to $-a \sqrt{3}$. However, the solution $V$ cannot be discontinuous at $\xi=\pi$; therefore, its representation should be changed by using the root $V_{2}$ or $V_{3}$ instead of $V_{1}$. In this case, from Eqs. (37) and (38), we choose the root $V_{2}$, which varies from $-a \sqrt{3}$ to $a \sqrt{3}$ at $\xi=\pi$, whereas $V_{3}=0$ at $\xi=\pi$. As a result, at $\xi=\pi$, the solution $V=$ $V_{1}(\xi)$ changes to $V=V_{2}(\xi)$ so as to remain continuous.

The latter representation is valid up to $\xi=\pi+\xi_{s}$. At $\xi=\pi+\xi_{S}$, the solution exhibits another jump from $V=$ $V_{2}\left(\pi+\xi_{S}\right)=a$ to $V=V_{1}\left(\pi+\xi_{S}\right)=-2 a$, which corresponds to the jump from point $E$ to point $F$.

As the argument increases further to $\xi=3 \pi / 2$, the solution $V=V_{1}(\xi)$ reaches its minimum, corresponding to point $G$ in Fig. 6:

$$
\begin{gathered}
V(3 \pi / 2)=V_{1}(3 \pi / 2) \\
=-\sqrt[3]{0.5+0.5 \sqrt{1-4 a^{6}}}-\sqrt[3]{0.5-0.5 \sqrt{1-4 a^{6}}} .
\end{gathered}
$$

Expression (40) differs from Eq. (39) only in sign.

When the increasing argument $\xi$ passes through the value $\xi=2 \pi$, the function $\sin \xi$ again changes sign, and the root $V_{1}$ varies from $-a \sqrt{3}$ to $a \sqrt{3}$. Since the solution should be continuous at $\xi=2 \pi$, it should be described not by the root $V_{1}(\xi)$, but by one of the other roots: $V_{2}(\xi)$ or $V_{3}(\xi)$. From Eqs. (37) and (38), we obtain that, for the argument $\xi$ increasing from the value $\xi=2 \pi$, the roots are equal to $V_{2}(2 \pi)=-a \sqrt{3}$ and $V_{3}(2 \pi)=0$.

Thus, to retain the continuity of the solution at the passage through the point $\xi=2 \pi$, the representation of the solution should be changed from $V_{1}(2 \pi-0)=-a \sqrt{3}$ to $V_{2}(2 \pi+0)=-a \sqrt{3}$. Note that the use of the root $V_{3}=0$ at the beginning of motion was an exception related to the choice of the initial condition inconsistent with the steady-state regime of oscillations. To describe the steady-state periodic motion, it is necessary to use another root and set $V(2 \pi)=V_{2}(2 \pi)=-a \sqrt{3}$.

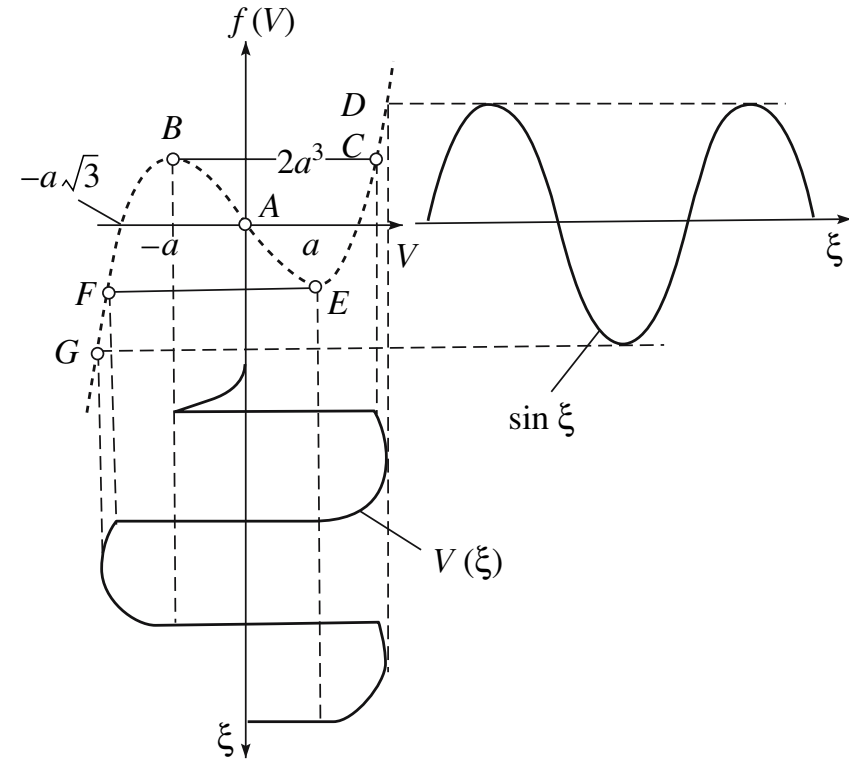

Fig. 6. Graphical analysis of the wave profile (by analogy with Fig. 5) for large positive values of detuning $\delta=j+a^{2}$ in the case of $2 a^{3}<1$.

The next discontinuity arises at $\xi=2 \pi+\xi_{S}$, where $V$ varies from $V=V_{2}\left(2 \pi+\xi_{S}\right)=-a$ to $V=V_{1}\left(2 \pi+\xi_{S}\right)=$ $2 a$. This jump was already considered for $\xi_{S}=\xi_{S}$, and, hence, the first period of oscillations of $V(\xi)$ is completely described.

Now, let us summarize the results of analyzing the solutions to Eq. (34) for the parameter satisfying the condition $2 a^{3}<1$.

(i) In the region $0 \leq \xi \leq \xi_{S}$ or $0 \leq \sin \xi \leq \sin \xi_{S}=2 a^{3}$, the solution is given by the formula

$$
\begin{gathered}
V=V_{3}(\xi)=-a \cos f(\xi)+\sqrt{3} a \sin f(\xi), \\
V_{3}(0)=0, \quad V_{3}\left(\xi_{S}\right)=-a .
\end{gathered}
$$

(ii) In the region $\xi_{S} \leq \xi \leq \pi-\xi_{S}, 2 a^{3} \leq \sin \xi \leq 1$, the solution is given by the formula

$$
\begin{gathered}
V=V_{1}=\sqrt[3]{0.5 \sin \xi+0.5 \sqrt{\sin ^{2} \xi-4 a^{6}}} \\
+\sqrt[3]{0.5 \sin \xi-0.5 \sqrt{\sin ^{2} \xi-4 a^{6}}} \\
V_{1}\left(\xi_{S}\right)=2 a,
\end{gathered}
$$

$$
\begin{gathered}
V_{1}(\pi / 2)=\sqrt[3]{0.5+0.5 \sqrt{1-4 a^{6}}}+\sqrt[3]{0.5-0.5 \sqrt{1-4 a^{6}}}, \\
V_{1}\left(\pi-\xi_{S}\right)=2 a .
\end{gathered}
$$

(iii) In the region $\pi-\xi_{S} \leq \xi \leq \pi, 0 \leq \sin \xi \leq 2 a^{3}$, the solution is given as

$$
\begin{gathered}
V_{1}=V_{1}(\xi)=2 a \cos f(\xi), \\
V_{1}\left(\pi-\xi_{S}\right)=2 a, \quad V_{1}(\pi)=a \sqrt{3} .
\end{gathered}
$$


(iv) In the region $\pi \leq \xi \leq \pi+\xi_{S},-2 a^{3} \leq \sin \xi \leq 0$, the solution is

$$
\begin{gathered}
V=V_{2}(\xi)=a \cos f(\xi)+\sqrt{3} a \sin f(\xi), \\
V_{2}(\pi)=a \sqrt{3}, \quad V_{2}\left(\pi+\xi_{s}\right)=a .
\end{gathered}
$$

(v) In the region $\pi+\xi_{S} \leq \xi \leq 2 \pi-\xi_{S},-1 \leq \sin \xi \leq$ $-2 a^{3}$, the solution is

$$
\begin{gathered}
V=V_{1}=-\sqrt[3]{0.5|\sin \xi|+0.5 \sqrt{\sin ^{2} \xi-4 a^{6}}} \\
-\sqrt[3]{0.5|\sin \xi|-0.5 \sqrt{\sin ^{2} \xi-4 a^{6}}}, \\
V_{1}\left(\pi+\xi_{S}\right)=-2 a, \\
V_{1}(3 \pi / 2)=-\sqrt[3]{0.5+0.5 \sqrt{1-4 a^{6}}} \\
-\sqrt[3]{0.5-0.5 \sqrt{1-4 a^{6}}}, \quad V_{1}\left(2 \pi-\xi_{s}\right)=-2 a .
\end{gathered}
$$

(vi) In the region $2 \pi-\xi_{S} \leq \xi \leq 2 \pi,-2 a^{3} \leq \sin \xi \leq 0$, the solution is

$$
\begin{gathered}
V=V_{1}(\xi)=-2 a \cos f_{1}(\xi), \quad f_{1}(\xi) \equiv \frac{1}{3} \arccos \frac{\sin \xi}{2 a^{3}}, \\
V_{1}\left(2 \pi-\xi_{S}\right)=-2 a, \quad V_{1}(2 \pi)=-a \sqrt{3} .
\end{gathered}
$$

(vii) In the region $2 \pi \leq \xi \leq 2 \pi+\xi_{S}, 0 \leq \sin \xi \leq 2 a^{3}$, the solution is

$$
\begin{gathered}
V=V_{2}(\xi)=-a \cos f(\xi)-\sqrt{3} a \sin f(\xi), \\
V_{2}(2 \pi)=-a \sqrt{3}, \quad V_{2}\left(2 \pi+\xi_{S}\right)=-a .
\end{gathered}
$$

(viii) In the region $2 \pi+\xi_{S} \leq \xi \leq 3 \pi-\xi_{S}$, the solution coincides with that obtained for region (ii).

Analyzing the behavior of the solution in the latter case, $j-\delta \equiv-a^{2}<0$ and $0<2 a^{3}<1$, we assumed that the function describing the wave profile contains discontinuities. The aforementioned compression shocks (jumps from $V=-a$ to $V=2 a$ ) and expansion shocks (jumps from $V=a$ to $V=-2 a$ ) can occur only when the positions of the imaging points $B$ and $E$ are unstable while its positions $C$ and $F$ are stable.

To analyze the stability, we consider Eq. (27) with allowance for the term containing the derivative and the small parameter $\Gamma$ :

$$
\Gamma \frac{d V}{d \xi}+V^{3}-3 a^{2} V=\sin \xi .
$$

Here, we have two time scales: the "slow" time $\xi$ and the "fast" time $\xi / \Gamma$. To move along the segment $B C$, the slow time should be "frozen": $\xi=\xi_{S}$ and $\sin \xi_{S}=$ $2 a^{3}$. Then, Eq. (48) takes the form

$$
\Gamma \frac{d V}{d \xi}=-(V+a)^{2}(V-2 a)
$$

To study the stability of point $B$, we set $V=-a+V^{\prime}$ in Eq. (49) and linearize this equation with respect to the small deviation $V$ :

$$
\Gamma \frac{d V^{\prime}}{d \xi}=3 a^{2} V^{\prime 2}
$$

The solution to this equation

$$
V^{\prime}=\frac{V^{\prime}(0)}{1-3 a V^{\prime}(0) \frac{\xi}{\Gamma}}
$$

shows that any positive initial perturbation $V^{\prime}(0)>0$ is a growing function of the fast time, and point $B$ is unstable. Leaving point $B$, the perturbation grows, and the system moves toward point $C$. Equation (49) shows that $C$ is an immobile point, because, at this point, $V=2 a$ and $d V / d \xi=0$. To investigate the stability of position $C$, we set $V=2 a+V^{\prime}$ in Eq. (49). The corresponding linearized equation and its solution have the form

$$
\Gamma \frac{d V^{\prime}}{d \xi}=-9 a^{2} V^{\prime}, \quad V^{\prime}=V^{\prime}(0) \exp \left(-9 a^{2} \frac{\xi}{\Gamma}\right)
$$

which demonstrates stability: any initial perturbation $V^{\prime}(0)$ decreases as the fast time increases. In a similar way, it is possible to prove the instability of point $E$ and the stability of point $F$, which justify the necessity of the second jump $E F$.

\section{THE RESONANCE CURVE FOR FORCED DISCONTINUOUS OSCILLATIONS}

Now, it is possible to analyze the frequency characteristic of a cubically nonlinear resonator for forced discontinuous oscillations by numerical methods using Eqs. (41)-(47). It is evident that the resonance curve for discontinuous waves should considerably differ from the quasi-linear characteristics that are calculated by the harmonic balance method and shown in Fig. 3.

We intend to publish the results of numerical calculations later. In this paper, we limit our consideration to the qualitative analysis of a simplified model problem in order to reveal the basic features. We take into account the complicated nonlinear behavior of the system that was analyzed above using Figs. 4-6.

To obtain analytical formulas, we proceed from the problem statement based on cubic equation (28) with additional conditions (29), i.e.,

$$
\begin{gathered}
f(V)=V^{3}+3(j-\delta) V=\sin \xi, \\
\langle V\rangle=0, \quad\left\langle V^{2}\right\rangle=j,
\end{gathered}
$$

to the "model" statement

$$
\begin{gathered}
f(V)=V|V|+2(j-\delta) V=\varphi(\xi), \\
\langle V\rangle=0, \quad\left\langle V^{2}\right\rangle=j .
\end{gathered}
$$


Here, the cubic parabola is approximated by two arcs of a quadratic parabola, while the sine on the righthand side of Eq. (53) is replaced by the function

$$
\begin{gathered}
\varphi(\xi)=1-\xi^{2}, \quad-1<\xi<1 ; \\
\varphi(\xi)=-1+(\xi-2)^{2}, \quad 1<\xi<3
\end{gathered}
$$

periodically extended with a period equal to 4 . Model (54), (55) allows a complete analytical solution. The analysis is performed below according to the scheme described in Section 5 with the same specific cases (a)-(d).

(a) Let the mean intensity exceed the detuning, i.e., $j-\delta \equiv a^{2}>0$.

In this case, within the half-period $-1<\xi<1$, Eq. (54) takes the form

$$
f(V)=V^{2}+2 a^{2} V=1-\xi^{2} .
$$

Averaging the solution to the quadratic equation (56), we obtain

$$
\left\langle V^{2}\right\rangle=j=\delta\left(a^{2}\right)+a^{2},
$$

where

$\delta\left(a^{2}\right)=a^{4}-a^{2}+\frac{2}{3}-a^{2}\left(1+a^{4}\right) \arcsin \left(1+a^{4}\right)^{-1 / 2}$.

(b) Let the mean intensity be equal to the detuning, i.e., $j-\delta=0$.

Equation (54) for $-1<\xi<1$ has the form

$$
f(V)=V^{2}=1-\xi^{2} .
$$

By averaging the solution, we obtain $\delta=j=2 / 3$.

(c) Let the positive detuning exceed the mean intensity, i.e., $j-\delta \equiv-a^{2}<0$, and, in addition, $a^{2}>1$.

For this case, Eq. (54) in the region $-1<\xi<1$ is as follows:

$$
f(V)=V^{2}-2 a^{2} V=1-\xi^{2} .
$$

Performing the necessary calculations, we arrive at the formula

$$
\delta\left(a^{2}\right)=a^{4}+a^{2}-\frac{2}{3}-a^{2}\left(a^{4}-1\right) \ln \sqrt{\frac{a^{2}+1}{a^{2}-1}} .
$$

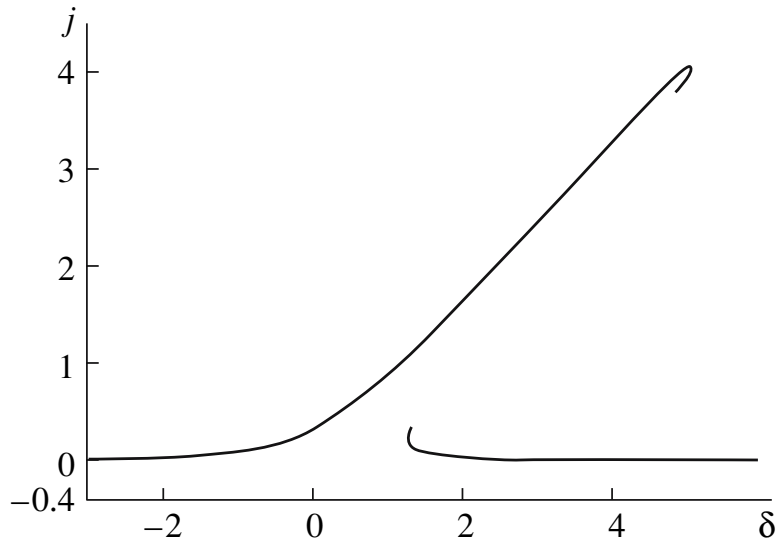

Fig. 7. Resonance curve for discontinuous oscillations in a cubically nonlinear acoustic resonator.

(d) Let the positive detuning exceed the mean intensity, i.e., $j-\delta \equiv-a^{2}<0$, but $a^{2}<1$.

By averaging the solution to Eq. (60) with allowance for the jumps illustrated in Fig. 6, we obtain the expression

$$
\begin{array}{r}
\delta\left(a^{2}\right)=3 a^{4}+a^{2}+\sqrt{1-a^{4}}\left[\frac{2}{3}+\left(\frac{1}{3}+\frac{\sqrt{2}}{2}\right) a^{4}\right] \\
+\frac{1}{2} a^{2}\left(1-a^{4}\right) \ln \sqrt{\frac{1-a^{2}}{1+a^{2}}} \\
+\frac{1}{2} a^{2}\left(1+a^{4}\right)\left[\arcsin \sqrt{\frac{1-a^{4}}{1+a^{4}}}+\arcsin \frac{1}{\sqrt{1+a^{4}}}\right] .
\end{array}
$$

The three branches of the resonance curve given by Eqs. (58), (61), and (62) are sewn together as shown in Fig. 7. Comparing the curves plotted in Figs. 7 and 3, we conclude that the frequency characteristic in Fig. 7 is the limiting curve for the characteristics shown in Fig. 3 in the case of viscosity tending to zero or the boundary oscillation amplitude tending to infinity. At the same time, the nonlinear absorption at the shock fronts prevails over the conventional linear absorption. As one can see from Fig. 7, the field intensity in the resonator cannot exceed a certain maximum value; i.e., a nonlinear saturation takes place.

The ambiguity observed in the dependence of the field intensity on the frequency detuning in Fig. 7 is presumably eliminated in the process of reaching the absolute maximum by smoothly increasing or decreasing the frequency of wall oscillations, by analogy with the forced oscillations in the Duffing equation model. The latter problem is described in many textbooks on the theory of oscillations and also considered in detail in [16]. If this analogy is valid, the maximum field intensity will be reached with an increase in the wall oscillation frequency; after the absolute maximum is 
reached, the intensity decreases in a jumplike manner to a certain small value. If we now reduce the frequency, the inverse jump from the lower branch to the upper one will occur at a smaller value of detuning $\delta$. Hence, a smooth variation in the frequency of large-amplitude wall oscillations leads to a hysteresis. In the region of detuning values where the hysteresis is present, a bistability associated with the aforementioned jumps is possible, and a transition from regular to chaotic field oscillations in the resonator may occur. However, this analogy may be inapplicable, because a resonator is a more complicated system that cannot be described by ordinary differential equations. To verify the validity of the aforementioned analogy and to reveal the features in the behavior of a resonator as a nonlinear distributed system, additional studies are necessary.

\section{CONCLUSIONS}

Thus, we proposed a method for analyzing intense oscillations of a planar acoustic resonator filled with a cubically nonlinear medium. We developed a theory that describes strongly distorted waves containing compression and expansion shock fronts. We determined the resonance characteristics for the case of linear absorption, as well as for very large amplitudes of boundary oscillations, at which the field in the resonator reaches saturation. We revealed the possibility of hysteresis and bistability in the system. The results of this study can be useful for biomedical and geophysical applications of nonlinear acoustics, as well as for problems of nonlinear diagnostics (primarily, shear elasticity measurements). Today, these applications are the object of active research.

\section{ACKNOWLEDGMENTS}

We are grateful to A.E. Sobisevich for useful discussions. This work was supported in part by the Russian Foundation for Basic Research (project no. 06-0216658) and the Presidential Program in Support of the
Leading Scientific Schools of Russia (grant no. NSh4449.2006.2).

\section{REFERENCES}

1. A. B. Coppens and A. A. Atchley, in Encyclopedia of Acoustics (Wiley, New York, 1997), pp. 237-246.

2. O. V. Rudenko, C. M. Hedberg, and B. O. Énflo, Akust. Zh. 47, 525 (2001) [Acoust. Phys. 47, 453 (2001)].

3. B. O. Enflo, C. M. Hedberg, and O. V. Rudenko, J. Acoust. Soc. Am. 117 (2), 601 (2005).

4. I. P. Lee-Bapty and D. G. Crighton, Philos. Trans. R. Soc. London, Ser. A 323, 173 (1987).

5. O. V. Rudenko and O. A. Sapozhnikov, Zh. Éksp. Teor. Fiz. 106, 395 (1994) [JETP 79, 220 (1994)].

6. E. A. Zabolotskaya, Akust. Zh. 32, 474 (1986) [Sov. Phys. Acoust. 32, 286 (1986)].

7. B. O. Enflo, C. M. Hedberg, and O. V. Rudenko, in Mathematical Modeling of Wave Phenomena, Växiö, Sweden, 2005, AIP Conf. Proc. 834, 187 (2006).

8. A. P. Sarvazyan, O. V. Rudenko, S. D. Swanson, et al., Ultrasound Med. Biol. 24, 1419 (1998).

9. S. Catheline, J.-L. Gennisson, and M. Fink, J. Acoust. Soc. Am. 114, 3087 (2003).

10. L. A. Ostrovsky and P. A. Johnson, Riv. Nuovo Cimento 24 (7), 1 (2001).

11. V. G. Andreev and T. A. Burlakova, Akust. Zh. 53, 50 (2007) [Acoust. Phys. 53, 44 (2007)].

12. V. V. Kaner, O. V. Rudenko, and R. V. Khokhlov, Akust. Zh. 23, 756 (1977) [Sov. Phys. Acoust. 23, 432 (1977)].

13. O. V. Rudenko and S. I. Soluyan, Theoretical Foundations of Nonlinear Acoustics (Nauka, Moscow, 1975; Consultants Bureau, New York, 1977).

14. O. V. Rudenko, Usp. Fiz. Nauk 165, 1011 (1995) [Phys. Usp. 38, 965 (1995)].

15. O. V. Rudenko and O. A. Sapozhnikov, Usp. Fiz. Nauk 174, 973 (2004) [Phys. Usp. 47, 973 (2004)].

16. A. P. Kuznetsov, S. P. Kuznetsov, and N. M. Ryskin, Nonlinear Oscillations (Fizmatlit, Moscow, 2002) [in Russian].

Translated by E. Golyamina 